\title{
Evaluation of the Effects of Different Rapid Maxillary Expansion Appliances on Obstructive Sleep Apnea: A randomized clinical trial
}

\author{
Gokcenur Gokce ${ }^{1}$, Ozen K Basoglu², and Ilknur Veli ${ }^{1}$ \\ ${ }^{1}$ Izmir Katip Celebi University \\ ${ }^{2}$ Ege University
}

July 12, 2021

\begin{abstract}
Purpose The aim of this randomized clinical trial was to evaluate the effects of tooth tissue-borne (TTB), tooth-borne (TB), and bone-borne (BB) rapid maxillary expansion appliances on obstructive sleep apnea (OSA) severity. Trial design Three-arm parallel randomized controlled trial. Methods This study was designed in parallel with an allocation ratio of 1:1:1. Forty six patients with narrow maxilla and diagnosis of OSA recruited from the Department of Orthodontics, \#\#\# University were randomly assigned to three groups according to appliance used: tooth tissue-borne, tooth-borne and bone-borne expanders. The primary outcome of this study included polygraphic change in sleep parameters. Secondary outcome was the correction of posterior crossbite. Each subject underwent overnight sleep test with polygraphy at baseline and 3 month-follow-up of treatment. Randomization was performed using a computer-generated randomization program The outcome assessor was blinded to group assignment. For the statistical analysis, Kruskal-Wallis analysis and Dunn-Bonferroni tests were used for intergroup comparisons and Wilcoxon analysis was used for intra-group evalaution. $\mathrm{p}<0.05$ was accepted statistically significant. Results The amount of expansion in maxillary width and upper intermolar width were similar in all goups (95\% confidence interval [CI], $\mathrm{p}>0.05$ ). The groups were similar in terms of apnea-hypopnea index (AHI) and oxygen saturation parameters at baseline (95\% [CI], p>0.05). After 3 months of treatment, there was no significant decrease in AHI and oxygen desaturation index, and no increase in minimum and mean oxygen saturations (95\% [CI], p >0.05). Supine AHI values were decreased by the tooth tissue-borne and tooth-borne appliances, but these changes were not significant (95\% [CI], p >0.05). Harms No serious harm ocurred except mild gingivitis. Conclusions Similar skeletal and dental expansion of maxilla were observed after RME with all expanders. Although the decrease in AHI was not significant, RME can be used as an adjunct to the primary treatment in OSA patients.
\end{abstract}

\section{Evaluation of the Effects of Different Rapid Maxillary Expansion Appliances on Obstructive Sleep Apnea: A randomized clinical trial}

\section{Abstract}

Purpose The aim of this randomized clinical trial was to evaluate the effects of tooth tissue-borne (TTB), tooth-borne (TB), and bone-borne (BB) rapid maxillary expansion appliances on obstructive sleep apnea (OSA) severity.

Trial design Three-arm parallel randomized controlled trial.

Methods This study was designed in parallel with an allocation ratio of 1:1:1. Forty six patients with narrow maxilla and diagnosis of OSA recruited from the Department of Orthodontics, \#\#\# University were randomly assigned to three groups according to appliance used: tooth tissue-borne, tooth-borne and bone-borne expanders. The primary outcome of this study included polygraphic change in sleep parameters. 
Secondary outcome was the correction of posterior crossbite. Each subject underwent overnight sleep test with polygraphy at baseline and 3 month-follow-up of treatment. Randomization was performed using a computer-generated randomization program The outcome assessor was blinded to group assignment. For the statistical analysis, Kruskal-Wallis analysis and Dunn-Bonferroni tests were used for inter-group comparisons and Wilcoxon analysis was used for intra-group evalaution. $p<0.05$ was accepted statistically significant.

Results The amount of expansion in maxillary width and upper intermolar width were similar in all goups (95\% confidence interval $[\mathrm{CI}], p>0.05)$. The groups were similar in terms of apnea-hypopnea index (AHI) and oxygen saturation parameters at baseline $(95 \%[\mathrm{CI}], p>0.05)$. After 3 months of treatment, there was no significant decrease in AHI and oxygen desaturation index, and no increase in minimum and mean oxygen saturations $(95 \%[\mathrm{CI}], p>0.05)$. Supine AHI values were decreased by the tooth tissue-borne and tooth-borne appliances, but these changes were not significant $(95 \%[\mathrm{CI}], p>0.05)$.

Harms No serious harm ocurred except mild gingivitis.

Conclusions Similar skeletal and dental expansion of maxilla were observed after RME with all expanders. Although the decrease in AHI was not significant, RME can be used as an adjunct to the primary treatment in OSA patients.

Trial Registration This study was registered at Clinicaltrials.gov (Identifier \#).

Protocol The protocol was not published.

Funding This trial was supported by \# University, Scientific Research Projects Unit [grant number \#].

\section{What's known}

Obstructive sleep apnea (OSA) is one of the most common sleep-related respiratory disorders generally accompanied with craniofacial anomalies. Many studies suggest that narrow maxilla may play a role in mouth breathing and OSA pathophysiology. RME is considered as a potential additional treatment in children with OSA. We evaluated and compared the effects of tooth tissue-borne (TTB), tooth-borne (TB), and bone-borne (BB) appliances on OSA severity in this randomized clinical trial.

\section{What's new}

Although it is stated in the literature that bone borne appliances are more effective in maxillary expansion,RME was found to be effective in OSA treatment independently of the appliance design. Although the decrease in the AHI value was not significant, RME can be applied as an adjunct to primary treatment in OSA patients.

\section{INTRODUCTION}

Obstructive sleep apnea (OSA) is one of the most common causes of sleep-related respiratory disorder associated with partial (hypopnea) or complete (apnea) upper airway obstruction occurring during sleep. ${ }^{1}$ Craniofacial anomalies are common in OSA patients. ${ }^{2,3}$ These abnormalities have a negative effect on upper airway dimensions and increase the risk of OSA. Many studies suggest that narrow maxilla may play a role in mouth breathing and OSA pathophysiology. ${ }^{4,5}$ Children with OSA have deep palate and maxillary hypoplasia with narrow maxilla. ${ }^{6-8}$ Maxillary constriction may also cause increase in nasal resistance, airflow decrease, and changes in tongue posture leading to OSA formation. ${ }^{9}$

Identification of the role of craniofacial abnormalities in the development of OSA has led to the emergence of some treatment strategies for the improvement of the craniofacial structure. Rapid maxillary expansion (RME) is a commonly used orthodontic technique in patients with maxillary width deficiency to provide skeletal expansion, correct posterior crossbite and position of the tongue and resolve naso-respiratory problems by reducing oral respiration. ${ }^{10-13}$ This method is based on the principle of opening the midpalatal suture under the effect of orthopedic forces that aims to achieve maximum bone effect and minimum dental effect in the maxillary complex. ${ }^{14}$ Since the maxilla is adjacent to many bones, the effects of RME are not limited to the maxilla. ${ }^{15}$ RME separates the outer walls of the nasal cavity laterally and causes lowering of the the 
palatal vault and the nasal septum to straight, reducing nasal resistance, increasing internal capacity, and improving breathing. ${ }^{15-17}$

Although there are many appliances used in RME treatment, for more controlled, more reliable and more efficient expansion of the maxilla, rigid and durable appliances are recommended. These appliances are generally divided into 2 as tooth borne and tooth tissue borne. ${ }^{18}$ Recently, with the development of intrabone anchor devices, the popularity of bone-borne maxillary expanders has increased. Bone-borne expanders with mini-screws ensure the direct transmission of force to the maxilla, thereby preventing the occurrence of side effects in conventional tooth-borne or tooth tissue-borne RME appliances. ${ }^{19}$

RME is considered as a potential additional treatment in children with OSA. ${ }^{13}$ The first study of the effects of RME on OSA was reported by Cistulli et al. ${ }^{4}$ Researchers reported that 9 of 10 patients with mild to moderate OSA had decreased apnea-hypopnea index (AHI) with RME. Pirelli et al ${ }^{10}$ showed that RME was an appropriate treatment for children with OSA without enlarged tonsils and adenoids, and $92.8 \%$ of children who had mouth breathing after RME had improvement and started nasal breathing as a result of the enlargement in the nasal cavity.

Specific objectives and hypotheses

To the best of the authors' knowledge, this was the first study to evaluate the effects of different designed RME appliances on OSA comparatively. Given that RME is used as an additional treatment method for mild-to-moderate OSA patients, the aim of this randomized clinical trial (RCT) was to evaluate and compare the effects of tooth tissue-borne (TTB), tooth-borne (TB), and bone-borne (BB) appliances on OSA severity. The null hypothesis was that there was no difference for the sleep apnea severity and amount of maxillary expansion among the expansion appliances.

\section{MATERIAL AND METHODS}

Trial design and any changes after trial commencement

This 3-arm parallel two-center study was a RCT and followed the 'Consolidated Standards of Reporting Trials' statement and principles ${ }^{20}$ and no methodological changes were required after the study started.

Participants, eligibility criteria, and settings

The study was approved by the Ethics Committee of Clinical Trials, \# University, \#, \# under protocol number \#. In addition, the protocol for this study with the identifier \# was registered in ClinicalTrials.gov. The patients were recruited in the department of Orthodontics, Faculty of Dentistry, University of \#\# from September 2016 to January 2018. The following eligibility criteria were applied: (1) no previous tonsillar, nasal or adenoid surgery and orthodontic treatment, (2) bilateral crossbite and need for RME, (3) maxillary and mandibular permanent teeth fully erupted, (4) AHI [?] 1 defined as OSA (21). Exclusion criteria were the presence of adenotonsillectomy or nasopharyngeal/oropharyngeal pathologies, craniofacial syndromes, body mass index (BMI) [?]30 (weight $(\mathrm{kg}) /$ height $\left(\mathrm{m}^{2}\right)$ ), ${ }^{22}$ systemic disease or poor oral hygiene. Thus, mouth breathing and factors that may cause OSA without maxillary constriction had been tried to be eliminated as much as possible. All patients were treated by the same clinician (\#) in the department of orthodontics and polygraphic data were obtained by the same sleep specialist (\#) in the sleep clinic.

Interventions

Before treatment, written consent forms which inform the patients about the orthodontic treatment, the necessary tests, the procedures to be performed and the complications that may occur were acquired from the patients and legal guardians. At this stage one investigator opened an envelope containing a card bearing the name of 1 of the 3 types of expansion appliances. Thus, the patients were divided into 3 groups randomly: TTB, TB and BB expanders (Figure 1, A-C).

All expansion appliances containing $9 \mathrm{~mm}$ Hyrax expansion screw (G\&Hß Orthodontics, United States) positioned parallel to the second premolars and were used to correct the posterior crossbite. In the TTB 
group, the occlusal and half of the palatinal and buccal surfaces of the molar and premolar teeth were coated with heat polymerized acrylic (Vertex Dental, Zeist, The Netherlands). Finally, the appliance was attached to posterior teeth with glass ionomer cement (Ketac Cem, 3M ESPE, Seefeld, Germany). In the TB group, long arms of the expansion screw are soldered to molar and premolar teeth bands. At last, the appliance was attached to posterior teeth with glass ionomer cement (Ketac Cem, 3M ESPE, Seefeld, Germany). In the BB group, two mini-screws (Tomas, Dentaurum, Germany) with a diameter of $1.6 \mathrm{~mm}$ and a length of 10 $\mathrm{mm}$ are located on the right and left sides between the roots of the 2nd premolar and 1st molar teeth with $60-70^{\circ}$ angle. The mini-screws were placed at a speed of 50 revolution per minute (rpm) with physio-dispenser under physiological saline irrigation. Then heat-polymerizing acrylic (Vertex Dental, Zeist, the Netherlands) was used to cover the screws and partially the palatal area. Consequently, the appliance was attached to the mini-screws in the palatinal area using the light-cured composite (Transbond XT, 3M Unitek, Monrovia, United States).

During RME, same expansion protocol was applied to all patients. The expansion appliance was activated one-quarter turn twice a day until the palatal cusp of the upper molar came into contact with the buccal cusp of the lower molar. After the desired expansion was achieved, the expansion screws were fixed with lightcuring band adhesive (Ultra Band-Lok, G\&H Orthodontics, USA). In order to calcification of midpalatal suture and the formation of new bone, appliances were left passively until the end of the 3 months retention phase.

Postero-anterior radiographs were obtained from each patients at baseline and 3 month-follow-up as a routine orthodontic record. These radiographs were analyzed in order to compare the treatment efficacy of RME appliances. Sleep tests were performed by polygraphy (SleepDoc Portiß)7, Dr. Fenyves \& Gut, Germany) at baseline and during 3 months follow-up after treatment with RME appliance. In addition to being easy to use in terms of patient and physician, this device basically provides 10-channel connections with two straps, a finger probe and a respiratory cannula. The patient and his / her parents were informed verbally that one night before the sleep test, they should not use caffeine or sedative medication before the application in order to record normal sleep rhythm during the test. The sleep technician explained how to attach and apply the device to the patient, and polygraphy was performed in the patient's home during spontaneous sleep in his or her own bed. Application of the device on the patient is shown in Figure 2. One-night sleep record data for each patient was transferred to the computer using the SleepDoc Porti $囚 7$ software package and a report was obtained.

Outcomes (primary and secondary) and any changes after trial commencement

The primary outcome of this study included polygraphic change in sleep parameters. Secondary outcome was the correction of posterior crossbite. Postero-anterior radiographs of 46 patients obtained at baseline and 3 month-follow-up were analyzed. In the reports obtained as a result of the overnight sleep test, the results were presented in 3 main sections; evaluation of breathing (apnea and hypopnea), pulse oximetry evaluation and position dependent evaluation. In our study, 7 parameters were evaluated: AHI $(/ \mathrm{h})$, number of apneas and hypopneas, oxygen desaturation index (ODI) (/h), minimum oxygen saturation (\%), mean oxygen saturation (\%) and supin AHI (/h).

After trial commencement there was no outcome changes occurred.

Sample size calculation

A power analysis established by $\mathrm{G}^{*}$ Power Version 3.1.3 (Franz Faul, Universität Kiel, Germany) software, sample size of 45 would give more than $83 \%$ (actual power $=0.8336062$ ) power to detect significant differences with 0.5 effect size and at the $\alpha=0.05$ significance level (critical $F=3.2199423$; noncentrality parameter $\lambda=11.2500000)$.

Interim analysis and stopping guidelines

Not inapplicable. 
Randomization (random number generation, allocation concealment, implementation)

Random Allocation Software program 2.0 was used to electronic randomization before trial beginning. Numbered, opaque sealed envelopes were prepared before the beginning of the trial in order to hide the allocation. Each envelope containing a card with the noun of 1 expansion appliance was opened sequentially. One operator specified information on the outer surface of the envelope, such as exhibitor initials, expander type and allocation date. The operator was responsible for random number generation, allocation concealment, implementation.

Blinding

It was not possible to blind the treating clinicians and patients during the trial because the clinician was aware of which type of expansion appliance was applied. However, blinding was accomplished during polygraphic data analysis. This data analysis was completed by sleep specialist (\#\#), who was blinded to any patient identifiable information or group allocation. Also, the statistician was also blind about group allocation.

Error study

15 postero-anterior radiographs were randomly selected and re-evaluated by the same researcher $(\mathrm{xx})$ after 3 -week interval. Dahlberg formula ${ }^{23}$ was used for the evaluation of method error. The intra-class correlation coefficients (ICCs) were used to quantify intraexaminer reliability.

Statistical analysis (primary and secondary outcomes, subgroup analyses)

IBM SPSS Statistics version 22.0 (SPSS Inc, Chicago, Ill) was used to perform the statistical analysis. Descriptive statistics were given as number of units (n), percentage (\%), mean \pm standard deviation $(\mathrm{x} \pm$ ss), median, 25th percentile (Q1) and 75th percentile (Q3). The normal distribution of the numerical variables was evaluated by Shapiro Wilk normality test and Q-Q graphs. Kruskal-Wallis analysis and Dunn-Bonferroni test were used for inter-group comparisons. Wilcoxon analysis was used for intra-group comparisons and $p$ $<0.05$ was considered statistically significant.

\section{RESULTS}

Participant flow

The CONSORT schema of the study (i.e., enrolment, intervention allocation, follow-up, and data analysis) is shown in (Figure 3). Fourty six patients were randomly allocated to be treated with TTB, TB and BB expanders. These groups included 15,15 and 16 patients, respectively. All patients randomized were analyzed in the trial without any dropout occurred.

\section{Baseline data}

Sixteen $(32.7 \%)$ of the patients included in the study were male and $30(67.3 \%)$ were female. TTB group contained 12 female and 3 male with a mean age of 12.5 years. TB group was composed of 6 female and 9 male with a mean age of 12.8 years. BB group included 13 female and 3 male with a mean age of 13.1 years. body mass index (BMI) (weight $(\mathrm{kg}) /$ height $\left(\mathrm{m}^{2}\right)$ ) values of groups were $22.2,22.8,23.2 \mathrm{~kg} / \mathrm{m}^{2}$, respectively. All patients had bilateral posterior crossbite. Postero-anterior analysis showed that all groups had similar upper intermolar width $(\mathrm{mm})$ and maxillary width $(\mathrm{mm})(95 \%(\mathrm{CI}), p>0.05$, Table 1$)$. The groups also showed similar characteristics concerning about variables of sleep (95\% (CI), $p>0.05$, Table 2). According to AHI classification, ${ }^{21} 16$ patients had mild, 13 patients had moderate and 17 patients had severe OSA at baseline of treatment.

\section{Numbers analyzed for each outcome, estimation and precision, subgroup analyses}

Errors for measurements ranged from $0.1 \mathrm{~mm}$ to $0.3 \mathrm{~mm}$, with a high degree of intraexaminer reliability noticed for all variables $(\mathrm{ICC}=0.956-0.985)$.

For the primary outcome included polygraphic assessment, data from 46 participants were analyzed at baseline and 3 month follow-up of treatment. There was no significant difference between the groups at 
baseline and 3 month follow-up in terms of AHI values (95\% (CI), $p>0.05$ ). Besides, AHI did not decrease significantly in any groups during 3-month follow-up with RME treatment (Table 3; Supplementary Figure 1 ). When we evaluated the number of apneas and hipopneas, no significant difference was found intra- and inter-groups during 3 months follow-up (95\% (CI), $p>0.05)$. There was a reduction in the number of apneas in the TTB and TB groups but the changes were not statistically significant (95\% (CI), $p>0.05)$. The value of supin AHI showing the position-based assessment of respiratory events were similar in three groups $(95 \%$ $(\mathrm{CI}), p>0.05)$. Supine AHI values were decreased non-significantly in the TTB and TB groups compared to the baseline (95\% (CI), $p>0.05)$ (Table 3$)$.

The difference between the groups in ODI $(/ \mathrm{h})$ was not statistically significant.There was a non-significant decrease in TB and BB groups after 3 months treatment (95\% (CI), $p>0.05$ ) (Table 3; Supplementary Figure 2). When the minimum and mean oxygen saturations were evaluated, there was no difference between the groups. In the $\mathrm{TB}$ and $\mathrm{BB}$ groups, a statistically insignificant increase in minimum oxygen saturation occurred at 3 month-follow-up of treatment (95\% (CI), $p>0.05$ ) (Table 3).

The secondary outcome of this study was the correction of posterior crossbite. 46 patients postero-anterior radiographs were analyzed at baseline and 3 month follow-up of treatment. There were no statistically significant differences between groups in the increase of upper intermolar width and maxillary width $(95 \%$ (CI), $p>0.05$ ) (Table 1).

\section{Harms}

There was no serious harm to oral health in the participants, except for mild gingivitis due to plaque accumulation.

\section{DISCUSSION}

\section{Main findings in the context of the evidence, interpretation}

To our knowledge, this is the first study designed to evaluate the effect of different RME appliances on OSA severity. This research may have a significant impact on clinical practice in terms of upper airway affects and general health.

OSA is a sleep-related breathing disorder characterized by recurrent full or partial obstruction (obstructive apnea or hypopnea) during sleep. ${ }^{24}$ The aims of the treatment of OSA are; prevention of apnea, hypopneas, desaturation and complications during sleep, alleviating symptoms and improving sleep quality. The relationship between OSA and maxillofacial malformations and malocclusions requires multidisciplinary approaches in treatment. ${ }^{13}$ This multidisciplinary team includes a sleep specialist, otolaryngologist, pediatrician, neurologist, psychiatrist, cardiologist and dentist. ${ }^{25}$

RME is an orthodontic treatment approach that has been used for years in children with maxillary constriction. ${ }^{24}$ During the treatment, while the skeletal expansion of the maxilla is ensured with orthopedic forces, it has also been reported that there is an increase in the volume of upper airway dimensions and decrease nasal resistency. ${ }^{24}$ Thus, RME may has the potential to play an important role in such a preventative treatment. In this multidisciplinary study, we aimed to evaluate the effect of RME treatment with different expanders on patients with maxillary constriction and OSA.

Each patient included in this multidisciplinary study was examined in the ENT clinic at the beginning of the treatment to determine whether the present snoring and mouth breathing were caused by a pathology (acute rhinitis, septum deviation, adenotonsillar hypertrophy, tumors, polyps) in the nasal or nasopharyngeal / oropharyngeal region. Patients with pathology were excluded from the study.

Obesity is one of the most important risk factors in the formation of OSA. ${ }^{26}$ The BMI of each patient was calculated at the beginning of the treatment, and patients in the obese class were not included in the study. Thus, the factors that may cause mouth breathing and OSA other than maxillary constriction were tried to be eliminated as much as possible. 
In many studies analyzing the effects of RME in the literature, the gender difference of individuals was not evaluated or this difference was not found to be significant. ${ }^{27,28}$ For this reason, gender distribution was not taken into account in our study.

Dental and skeletal classification in the sagittal direction were not made in the selection of individuals in the study similar to the RME studies in the literature. ${ }^{29,30}$ Kitagaito $^{29}$ performed a study to describe dentofacial characteristics (overjet, overbite, posterior crossbite, facial convexity) in children referred to a pediatric sleep center for OSA. Intraorally, Angle classification, overjet, overbite, and posterior crossbites were evaluated in mild, moderate, severe OSA groups with no OSA group. In conclusion, the mild and moderate to severe OSA groups did not exhibit statistically significant differences between no OSA group in Angle's classification. Similarly, Villa et al. ${ }^{30}$ stated no difference according to type of malocclusion among patients with OSA.

According to the results of polygraphic sleep records, 7 parameters (AHI, number of apnea and hypopnea, desaturation index, lowest desaturation, average saturation and supin AHI) were examined. The most commonly used parameter for determining the severity of OSA and respiratory events is AHI. ${ }^{21}$ AHI refers to the total number of apneas and hypopneas per hour during sleep. It is considered as abnormal if the value is more than 1 hour per hour in children. Patients with AHI values between 1-4.9 were classified as mild OSA, 5 to 9.9 cases as moderate OSA and $10>$ cases as severe OSA. ${ }^{21}$ According to this classification, 16 patients had mild, 13 patients had moderate and 17 patients had severe OSA at the beginning of the treatment in this study. When the AHI changes according to the treatment were evaluated, it was found that there was a decrease in the TTB and BB groups but this was not significant. Due to the changes in AHI values, it was observed that 5 cases initially included in the severe OSA class were classified as moderate OSA and 6 cases were classified as mild OSA after treatment.

There are many studies ${ }^{4,31,32}$ conducted in the literature to evaluate the effect of RME on OSA. When the results of these studies were examined, they were consistent with the results of our study partially. However, the decrease in the AHI, which did not reach statistical significance in our study, was found to be significant in many studies. We think that this difference in the results is due to the fact that small sample size and the initial AHI values of the patients were not high enough. Cistulli et al. ${ }^{4}$ investigated the effects of RME on OSA. They included individuals with moderate and severe OSA and evaluated the PSG results of individuals after treatment and reported that the majority of patients had reduced snoring and a significant decrease in AHI in all patients and no change in AHI value in only 1 patient. Caprioglio et al. ${ }^{31}$ concluded that RME treatment with Haas appliance resulted in a significant decrease in AHI. Villa et al. ${ }^{32}$ indicated that RME caused a significant decrease in AHI and the results obtained were stable for a long time. In another 12-month follow-up study made by Villa et al., ${ }^{11}$ a significant decrease in AHI was reported after treatment of patients with OSA with Hyrax. Pirelli et al. ${ }^{10}$ applied RME to 32 patients with a mean age of 8.7 years with maxillary constriction and OSA. The authors reported that the value of AHI, which was 12.2 before treatment decreased to less than 1 after 4 months of treatment and remained stable for 12 months. Therefore, they argued that RME could be used as an adjunct in the treatment of OSA. Miano et al. ${ }^{12}$ reported an increase in sleep duration with a significant decrease in AHI one year after RME treatment in children with OSA. Fastuca et al. ${ }^{33}$ observed a significant decrease in AHI with increase of upper, middle and lower airway volumes after RME treatment in OSA cases. Palmisona et al. ${ }^{34}$ reported that AHI decreased with oral breathing, snoring and sleepiness due to the $8.5 \mathrm{~mm}$ expansion of the maxilla with RME treatment. Taddei et al. ${ }^{35}$ reported that RME treatment was effective in preventing OSA development. Camacho et al. ${ }^{9}$ observed RME treatment led to a decrease in AHI in children. Similarly, Singh et al. ${ }^{36}$ reported less snoring with an average $80 \%$ reduction in AHI with maxiller expansion.

The changes in the number of apnea and hypopnea were not significant after treatment in all groups. There was an insignificant decrease in the number of apneas in the TTB and TB groups and in the number of hypopneas in all groups. Similar results were obtained by Rose and Schessl. ${ }^{37}$ Researchers reported that clinical symptoms improved and the number of apneas and snoring decreased during sleep after treatment with TTB.

The change in ODI was not statistically significant in all groups. ODI (/hr) increased after treatment in 
TTB group, but decreased in TB and BB groups. Similar results were obtained by Vinha et al. (38) and it was reported that daytime sleepiness may improve with a decrease in ODI after surgically assisted rapid maxillary expansion (SARME) in patients with OSA. Ashok et al. ${ }^{39}$ reported that after RME treatment with a bonded appliance, sleep efficiency and total sleep duration increased, and ODI and arousal number decreased in children, consistent with other studies.

There was no significant difference between the groups in the minimum oxygen saturation and mean oxygen saturation parameters. Similar results were obtained by Miano et al. ${ }^{12}$ The researchers reported no change in oxygen saturation after RME treatment in children with OSA. In contrast to our study, Caprioglio et al. ${ }^{31}$ reported an increase in oxygen saturation after RME. Villa et al. ${ }^{32}$ and Fastuca et al. ${ }^{33}$ observed an improvement in clinical symptoms with increased oxygen saturation after RME. Kim ${ }^{40}$ reported that oxygen saturation from $60 \%$ at baseline increased to $93 \%$ immediately after treatment in children with OSA. Camacho et al. ${ }^{9}$ and Singh et al. ${ }^{36}$ reported an improvement in oxygen saturation after RME in accordance with other studies.

The effect of RME treatment with different appliances on OSA was not investigated in detail in any study by polygraphy. Most of the RME studies reported improvement in the majority of sleep parameters, similar to our study. ${ }^{4,10}$ However, the data we obtained from our study showed that this improvement was not statistically significant. This may be due to the low sample size in our study and the lower values of sleep parameters in the pediatric population compared to adults. These are the limitations of the study.

\section{Limitations}

The reason for the lack of statistical significance of the results may be that the values of sleep parameters of the pediatric population are lower than adults and small sample size. In addition, sleep cannot be evaluated with polygraphy as comprehensively as PSG. These issues constitute the limitation of our study.

\section{Generalizability}

The generalizability of this RCT may be limited due to conducted in a single population and center.

\section{CONCLUSIONS}

The null hypothesis was accepted. The following results were obtained:

RME was found to be effective in OSA treatment independently of the appliance design.

Although the decrease in the AHI value was not significant, RME can be applied as an adjunct to primary treatment in OSA patients.

\section{Acknowledgements}

We thank to associate professor Dr. \#\# for his support with the statistical analysis.

\section{Conflict of interest}

The authors declare that they have no competing interests. The authors are responsible for the writing and content of this article.

\section{Ethics}

The study was approved by the Ethics Committee of Clinical Trials, \# University, \#, \# under protocol number \#\#.

\section{Funding}

This work was supported by \#\# University, Scientific Research Projects Unit (grant number \#\#\#).

Authors' contributions 
\#\#: Contributed to the study's conception and design, acquisition, analysis, and interpretation of data, contributed during the article elaboration process and final approval of the version to be published. \#\#\#: Contributed to the study's conception and design, acquisition and final approval of the version to be published. \#\#: Contributed to the study's conception and design, acquisition and final approval of the version to be published.

\section{References}

1. Toraldo DM, Di Michele L, Ralli M, Arigliani M, Passali GC, De Benedetto M, et al. Obstructive sleep apnea syndrome in the pediatric age: the role of the pneumologist. Eur Rev Med Pharmacol Sci 2019;23:15-8.

2. Vieira BB, Itikawa CE, De Almeida LA, Sander HS, Fernandes RM, Anselmo-Lima WT, et al. Cephalometric evaluation of facial pattern and hyoid bone position in children with obstructive sleep apnea syndrome. Int J Pediatr Otorhinolaryngol 2011;75: 383-6.

3. Zhang C, He H, Ngan P. Effects of twin block appliance on obstructive sleep apnea in children: a preliminary study. Sleep Breath 2013;17:1309-14.

4. Cistulli PA, Palmisano RG, Poole MD. Treatment of obstructive sleep apnea syndrome by rapid maxillary expansion. Sleep 1998;21:831-5.

5. Sánchez-Súcar AM, Sánchez-Súcar FB, Almerich-Silla JM, Paredes-Gallardo V, Montiel-Company JM, García-Sanz V, et al. Effect of rapid maxillary expansion on sleep apnea-hypopnea syndrome in growing patients. A meta-analysis. J Clin Exp Dent 2019;11:759-67.

6. Brockbank JC. Update on pathophysiology and treatment of childhood obstructive sleep apnea syndrome. Paediatr Respir Rev 2017;24:21-3.

7. Burghard M, Brożek-Madry E, Krzeski A. Sleep disordered breathing in children-diagnostic questionnaires, comparative analysis. Int J Pediatr Otorhinolaryngol 2019;120:108-11.

8. Pirelli P, Fanucci E, Giancotti A, Di Girolamo M, Guilleminault C. Skeletal changes after rapid maxillary expansion in children with obstructive sleep apnea evaluated by low-dose multi-slice computed tomography. Sleep Med 2019;60:75-80.

9. Camacho M, Chang ET, Song SA, Abdullatif J, Zaghi S, Pirelli P, et al. Rapid maxillary expansion for pediatric obstructive sleep apnea: A systematic review and meta-analysis. Laryngoscope 2017;127:1712-9.

10. Pirelli P, Saponara M, Guilleminault C. Rapid maxillary expansion in children with obstructive sleep apnea syndrome Sleep 2004;27:761-6.

11. Villa MP, Malagola C, Pagani J, Montesano M, Rizzoli A, Guilleminault C, et al. Rapid maxillary expansion in children with obstructive sleep apnea syndrome: 12-month follow-up. Sleep Med 2007;8:128-34.

12. Miano S, Rizzoli A, Evangelisti M, Bruni O, Ferri R, Pagani J, et al. NREM sleep instability changes following rapid maxillary expansion in children with obstructive apnea sleep syndrome. Sleep Med 2009;10:471-8.

13. Katyal V, Pamula Y, Daynes CN, Martin J, Dreyer CW, Kennedy D, et al. Craniofacial and upper airway morphology in pediatric sleep-disordered breathing and changes in quality of life with rapid maxillary expansion Am J Orthod Dentofacial Orthop 2013;144:860-71.

14. Weissheimer A, De Menezes LM, Mezomo M, Dias DM, De Lima EMS, Rizzatto SMD. Immediate effects of rapid maxillary expansion with Haas-type and hyrax-type expanders: a randomized clinical trial. Am J Orthod Dentofacial Orthop 2011;140:366-76.

15. Ghoneima A, Abdel-Fattah E, Hartsfield J, El-Bedwehi A, Kamel A, Kula K. Effects of rapid maxillary expansion on the cranial and circummaxillary sutures. Am J Orthod Dentofacial Orthop 2011;140:5109.

16. Del Rosso E, Farronato M, Giannini L. RME: Effects on the nasal septum. A CBCT evaluation. Eur J Paediatr Dent 2019;20:123.

17. Caldas LD, Takeshita WM, Machado AW, Bittencourt MAV. Effect of rapid maxillary expansion on nasal cavity assessed with cone-beam computed tomography. Dental Press J Orthod 2020;25:39-45. 
18. McNamara JA, Brudon WL. Orthodontics and orthopedic treatment in the mixed dentition. Ann Arbor, Needham Press; 1993.

19. Lagravère MO, Carey J, Heo G, Toogood RW, Major PW. Transverse, vertical, and anteroposterior changes from bone-anchored maxillary expansion vs traditional rapid maxillary expansion: a randomized clinical trial. Am J Orthod Dentofacial Orthop 2010;137:304-5.

20. Moher D, Schulz KF, Altman D, Consort Group. The CONSORT statement: revised recommendations for improving the quality of reports of parallel-group randomized trials. Jama 2001;285:1987-91.

21. Benedek P, Balakrishnan K, Cunningham MJ, Friedman NR, Goudy SL, Ishman SL, Lee GS. International Pediatric Otolaryngology group (IPOG) consensus on the diagnosis and management of pediatric obstructive sleep apnea (OSA). Int J Pediatr Otorhinolaryngol 2020;138:110276.

22. Tomlinson DJ, Erskine RM, Morse CI, Onambélé GL. Body fat percentage, body mass index, fat mass index and the ageing bone: their singular and combined roles linked to physical activity and diet. Nutrients 2019;11:195.

23. Dahlberg G. Statistical methods for medical and biological students. New York: Interscience Publications; 1940. p. 122-32.

24. Carroll JL. Obstructive sleep-disordered breathing in children: new controversies, new directions. Clin Chest Med 2003;24:261-82.

25. Luzzi V, Ierardo G, Di Carlo G, Saccucci M, Polimeni A. Obstructive sleep apnea syndrome in the pediatric age: the role of the dentist. Eur Rev Med Pharmacol Sci 2019;23:9-14.

26. Arens R, Muzumdar H. Childhood obesity and obstructive sleep apnea syndrome. J Appl Physiol 2010;108:436-44.

27. Noussios G, Skouras A, Chouridis P, Argyriou N, Gougousis S, Kontzoglou G. The use of acoustic rhinometry for the assessment of adenoid hypertrophy: a clinical study. Acta Otorrinolaringol Eng 2008;59:433-7.

28. Wolfson AR, Carskadon MA. Sleep Schedules and Daytime Functioning in Adolescents. Child Dev 1998;69:875-87.

29. Kitagaito K. Malocclusion in Pediatric Obstructive Sleep Apnea Refractory to Adenotonsillectomy Referred for Sleep Study (Doctoral dissertation); 2020.

30. Villa MP, Rizzoli A, Rabasco J, Vitelli O, Pietropaoli N, Cecili M, Malagola C. Rapid maxillary expansion outcomes in treatment of obstructive sleep apnea in children. Sleep Med 2015;16:709-16.

31. Caprioglio A, Meneghel M, Fastuca R, Zecca PA, Nucera R, Nosetti L. Rapid maxillary expansion in growing patients: correspondence between 3-dimensional airway changes and polysomnography. Int J Pediatr Otorhinolaryngol 2014;78:23-7.

32. Villa MP, Rizzoli A, Miano S, Malagola C. Efficacy of rapid maxillary expansion in children with obstructive sleep apnea syndrome: 36 months of follow-up. Sleep Breath 2011;15:179-84.

33. Fastuca R, Perinetti G, Zecca PA, Nucera R, Caprioglio A. Airway compartments volume and oxygen saturation changes after rapid maxillary expansion: a longitudinal correlation study. Angle Orthod 2015;85:955-61.

34. Palmisano RG, Wilcox I, Sullivan CE, Cistulli PA. Treatment of snoring and obstructive sleep apnoea by rapid maxillary expansion. Aust N Z J Med 1996;26:428-9.

35. Taddei M, Alkhamis N, Tagariello T, D'Alessandro G, Mariucci EM, Piana G. Effects of rapid maxillary expansion and mandibular advancement on upper airways in Marfan's syndrome children: a home sleep study and cephalometric evaluation. Sleep Breath 2015;19:1213-20.

36. Dave Singh G, Callister JD. Effect of a maxillary appliance in an adult with obstructive sleep apnea: a case report. Cranio 2013;31:171-5.

37. Rose E, Schessl J. Orthodontic procedures in the treatment of obstructive sleep apnea in children. J Orofac Orthop 2006;67:58-67.

38. Vinha PP, Eckeli AL, Faria AC, Xavier SP, de Mello-Filho FV. Effects of surgically assisted rapid maxillary expansion on obstructive sleep apnea and daytime sleepiness. Sleep Breath 2016;20:501-8.

39. Ashok N, Varma NS, Ajith VV, Gopinath S. Effect of rapid maxillary expansion on sleep characteristics in children. Contemp Clin Dent 2014;5:489-94. 
40. Kim M. Orthodontic treatment with rapid maxillary expansion for treating a boy with severe obstructive sleep apnea. Sleep Med Res 2014;5:33-6.

Table 1. Descriptive statistics and intergroup differences of the RME appliances

\begin{tabular}{llllll}
\hline & & GROUPS* & GROUPS* & GROUPS* \\
\hline \multirow{3}{*}{ Upper Intermolar Width $(\mathrm{mm})$} & $\mathrm{B} / \mathrm{L}$ & Tooth tissue-borne & Tooth-borne & Bone-borne & $P$ \\
& $\mathrm{~F} / \mathrm{U}$ & $42.60(40.99-44.21)$ & $44.83(42.56-47.10)$ & $42.69(40.21-45.16)$ & 0.187 \\
& $\mathrm{~F} / \mathrm{U}-\mathrm{B} / \mathrm{L}$ & $5.04(1.79-8.29)$ & $3.15(-1.10-7.41)$ & $5.41(3.46-7.35)$ & 0.560 \\
Maxillary Width $(\mathrm{mm})$ & $\mathrm{B} / \mathrm{L}$ & $49.03(47.36-50.71)$ & $49.25(47.49-51.00)$ & $49.04(47.21-50.88)$ & 0.978 \\
& $\mathrm{~F} / \mathrm{U}$ & $53.29(50.25-56.33)$ & $53.98(51.73-56.23)$ & $53.53(51.55-55.51)$ & 0.911 \\
& $\mathrm{~F} / \mathrm{U}-\mathrm{B} / \mathrm{L}$ & $4.25(1.51-7.00)$ & $4.74(1.67-7.81)$ & $4.49(2.23-6.74)$ & 0.963 \\
\hline
\end{tabular}

* Data are given as mean (95\% confidence intervals)

B/L, Baseline; F/U, Follow Up

Table 2. Baseline polygraphy measurements

\begin{tabular}{|c|c|c|c|c|c|}
\hline & Baseline (B/L) & Baseline (B/L) & Baseline $(\mathrm{B} / \mathrm{L})$ & Baseline (B/L) & Baseline $(\mathrm{B} / \mathrm{L})$ \\
\hline GROUPS & AHI & ODI & $\begin{array}{l}\text { Minimum } \\
\text { oxygen } \\
\text { saturation }\end{array}$ & $\begin{array}{l}\text { Mean oxygen } \\
\text { saturation }\end{array}$ & Supin AHI \\
\hline $\begin{array}{l}\text { Tooth } \\
\text { tissue-borne }\end{array}$ & $\begin{array}{l}10.08 \\
(6.84-13.33)\end{array}$ & $7.44(3.55-11.33)$ & $\begin{array}{l}80.53 \\
(75.79-85.26)\end{array}$ & $\begin{array}{l}96.46 \\
(95.91-97.01)\end{array}$ & $2.89(1.58-4.20)$ \\
\hline Tooth-borne & $7.71(4.07-11.35)$ & $6.88(4.38-9.39)$ & $\begin{array}{l}80.40 \\
(75.00-85.79)\end{array}$ & $\begin{array}{l}96.73 \\
(96.34-97.12)\end{array}$ & $1.96(1.44-2.48)$ \\
\hline Bone-borne & $7.38(5.02-9.75)$ & $5.86(4.50-7.23)$ & $\begin{array}{l}76.87 \\
(70.87-82.87)\end{array}$ & $\begin{array}{l}96.87 \\
(96.40-97.34)\end{array}$ & $1.70(1.22-2.19)$ \\
\hline$P$ & 0.366 & 0.674 & 0.511 & 0.423 & 0.096 \\
\hline
\end{tabular}

Data are given as mean (95\% confidence intervals)

AHI, Apnea Hypopnea Index; ODI, Oxygen Desaturation Index

\begin{tabular}{lllll}
\hline & Groups & Groups & Groups & Groups \\
\hline & Tooth tissue-borne & Tooth tissue-borne & Tooth tissue-borne & Tooth-bo \\
Sleep Parameters & B/L & F/U & p & B/L \\
AHI & 9,2 & 8 & 0,280 & 5,4 \\
ODI (/hr) & 5,2 & 5,6 & 0,977 & 6,2 \\
Minimum oxygen saturation (\%) & 82 & 80 & 0,401 & 81 \\
Mean oxygen saturation (\%) & 96 & 97 & 0,083 & 97 \\
Supin AHI (/hr) & 8 & 7 & 0,581 & 7 \\
\hline
\end{tabular}

Table 3. Effect of the rapid maxillary expansion appliances on sleep parameters

Data were given as median

Abbrevations: AHI, apnea hypopnea index; B/L, baseline; F/U, follow up; ODI, oxygen desaturation index 


\section{Figure Legends}

Figure 1 (A-C). RME appliances (A) Tooth tissue-borne (B) Tooth-bone (C) Bone-borne

Figure 2 Application of the polygraphy on the patient

Figure 3 CONSORT flow diagram

Supplementary Figure 1 Change in apnea-hypopnea index by rapid maxillary expansion appliances

Supplementary Figure 2 Change in oxygen desaturation index by rapid maxillary expansion appliances

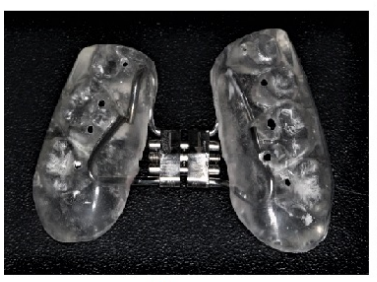

A

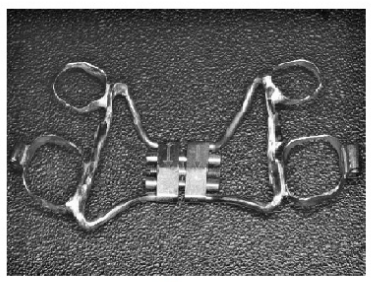

B

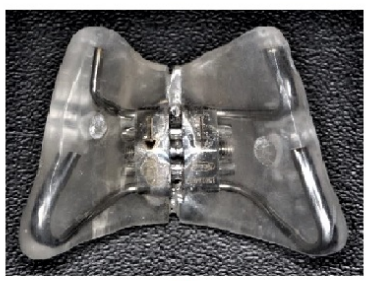

C

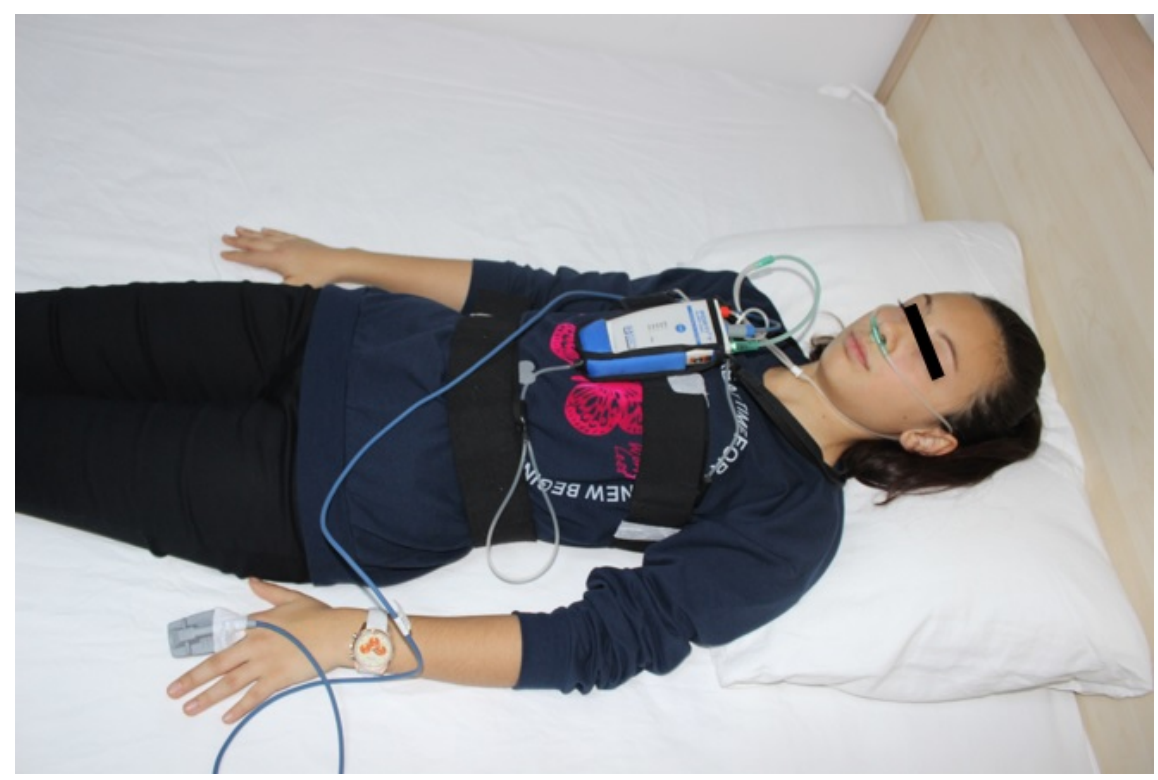




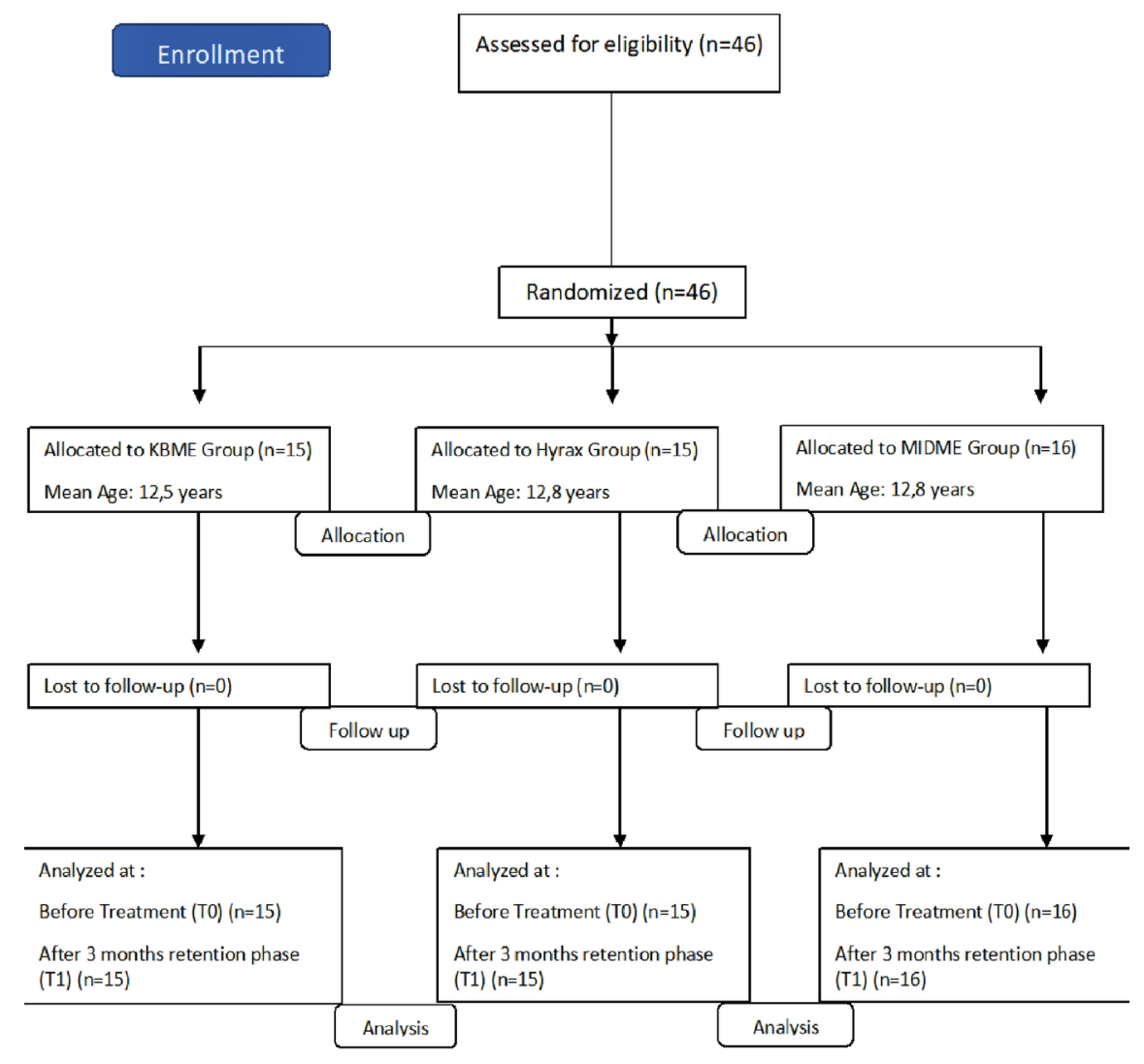

\section{Hosted file}

Table 1.docx available at https://authorea.com/users/425187/articles/530053-evaluationof-the-effects-of-different-rapid-maxillary-expansion-appliances-on-obstructive-sleepapnea-a-randomized-clinical-trial

\section{Hosted file}

Table 2.docx available at https://authorea.com/users/425187/articles/530053-evaluationof-the-effects-of-different-rapid-maxillary-expansion-appliances-on-obstructive-sleepapnea-a-randomized-clinical-trial

\section{Hosted file}

Table 3.docx available at https://authorea.com/users/425187/articles/530053-evaluationof-the-effects-of-different-rapid-maxillary-expansion-appliances-on-obstructive-sleepapnea-a-randomized-clinical-trial 


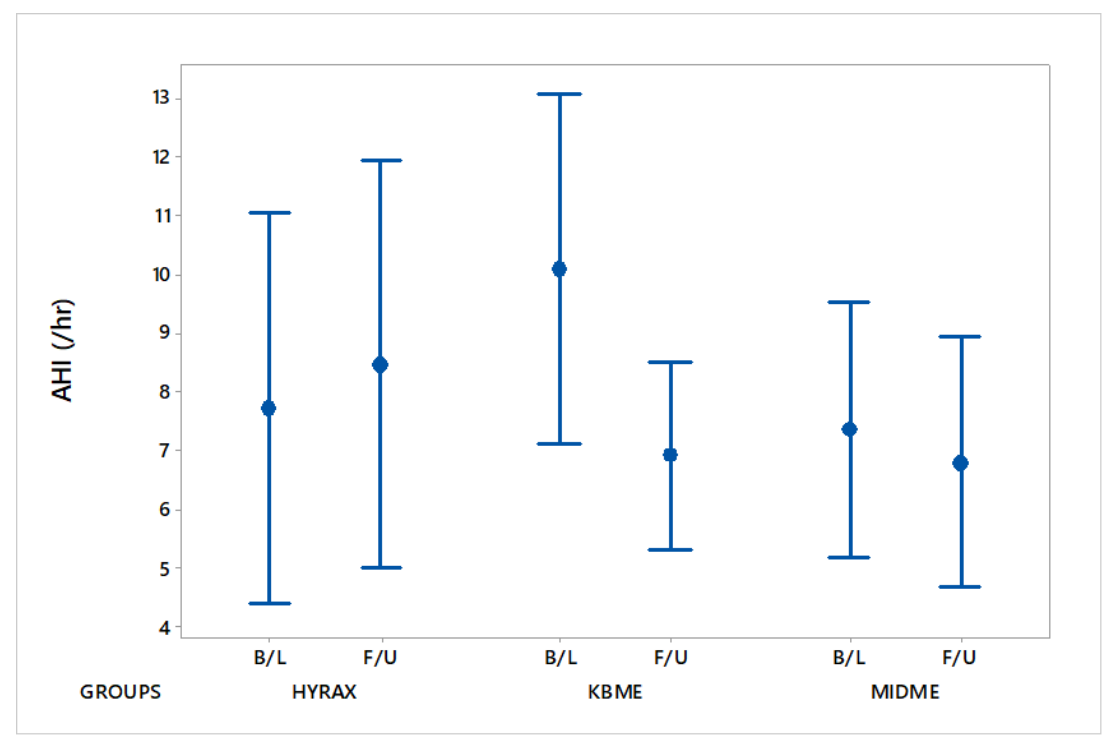

Error bars: 95\% Cl

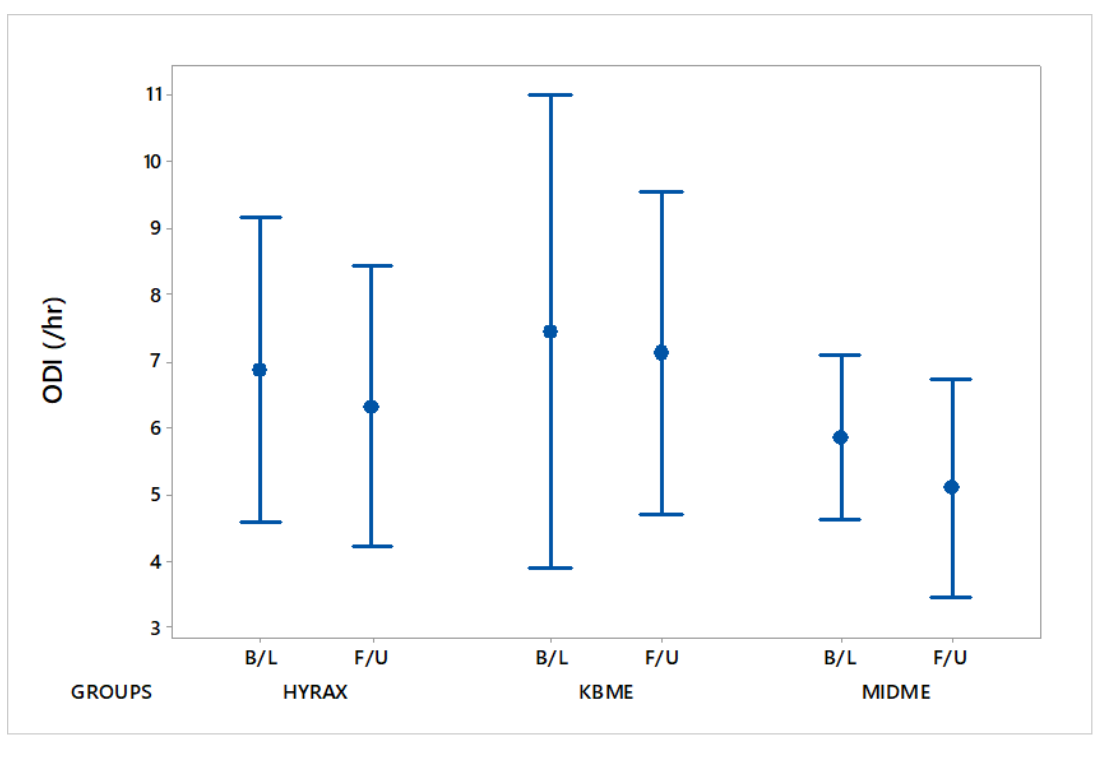

Error bars: $95 \% \mathrm{Cl}$ 